\title{
Sur les Problèmes Mixtes pour l’Équation des Ondes
}

\author{
Par
}

\section{Mitsuru IKAwA*}

\section{$\S 1$. Introduction}

Soit $\Omega$ un domaine de $\mathbf{R}^{n}=\left\{\left(x_{1}, \ldots, x_{n}\right) ; x_{i} \in \mathbf{R}\right\}$ dont la frontière $S$ est compacte et indéfiniment différentiable. Considérons le problème mixte

$$
\begin{cases}\square u(x, t)=\frac{\partial^{2} u}{\partial t^{2}}-\sum_{i=1}^{n} \frac{\partial^{2} u}{\partial x_{i}^{2}}=f(x, t) & \text { dans } \Omega \times 70, T[ \\ B u(x, t)=\frac{\partial u}{\partial v}+d(x) \frac{\partial u}{\partial t}=g(x, t) & \text { sur } S \times] 0, T[ \\ u(x, 0)=u_{0}(x) & \\ \frac{\partial u}{\partial t}(x, 0)=u_{1}(x) & \end{cases}
$$

où $\frac{\partial u}{\partial v}=\sum_{i=1}^{n} v_{i}(x) \frac{\partial u}{\partial x_{i}}$ et $v(x)=\left(v_{1}(x), v_{2}(x), \ldots, v_{n}(x)\right)$ est un vecteur réel indéfiniment différentiable défini sur $S$. On suppose que $v(x)$ est toujours transversal par rapport à $S$, c'est-à-dire, si l'on désigne la normale extérieure unitaire de $S$ par $n(x)=\left(n_{1}(x), n_{2}(x), \ldots, n_{n}(x)\right)$ il a lieu

$$
v(x) n(x)=\sum_{i=1}^{n} v_{i}(x) n_{i}(x) \neq 0 \quad \text { sur } \quad S
$$

Est-ce que la condition (1.2) serait suffisante, si l'on prend $d(x) \equiv 0$, pour que le problème mixte (1.1) soit bien posé au sens de $C^{\infty}$ ?

Communiqué par S. Matsuura, le 15 juin 1974.

* Département de Mathématique, Université d'Osaka, Osaka. 
Ici, on dit que le problème mixte (1.1) est bien posé au sens de $C^{\infty}$ quand pour toutes les données $\left\{u_{0}, u_{1}, f, g\right\}$ satisfaisant à la condition de compatibilité d'ordre infini (1.1) admet une solution unique $u$ dans $C^{\infty}(\bar{\Omega} \times[0, T])$ et pour chaque $t_{0} \in[0, T]$ l'application $\left\{u_{0}, u_{1}, f, g\right\} \rightarrow u$ est continue de $C^{\infty}(\bar{\Omega}) \times C^{\infty}(\bar{\Omega}) \times C^{\infty}\left(\bar{\Omega} \times\left[0, t_{0}\right]\right) \times C^{\infty}\left(S \times\left[0, t_{0}\right]\right)$ dans $C^{\infty}\left(\bar{\Omega} \times\left[0, t_{0}\right]\right)$.

La condition de compatibilité d'ordre infini pour les données $\left\{u_{0}, u_{1}\right.$, $f, g\}$ signifie qu'il a lieu

$$
\frac{\partial u_{p}}{\partial v}+d(x) u_{p+1}=\frac{\partial^{p} g}{\partial t^{p}}(x, 0) \quad \text { sur } S
$$

pour $p=0,1,2, \ldots$, où $u_{p}(x), p=2,3, \ldots$ sont définies par récurrence par la formule

$$
u_{p}(x)=\Delta u_{p-2}(x)+\frac{\partial^{p-2} f}{\partial t^{p-2}}(x, 0)
$$

A propos de la question posée tout à l'heure, ou de savoir si le problème mixte (1.1) est bien posé au sens de $C^{\infty}$ sous les conditions de $d(x) \equiv 0$ et de (1.2), la réponse est négative en général. Nous avons montré dans [4] et [5] que, au cas de $n=2$, on peut trouver un exemple d'un domaine $\Omega$ et d'un vecteur réel $v(x)$ défini sur $\partial \Omega$ tel que le problème mixte (1.1) n'est pas bien posé au sens de $C^{\infty}$.

Le domaine $\Omega$ construit dans [5] est convexe, compact et à la frontière indéfiniment différentiable. La méthode de construction de $\Omega$ et de $v(x)$ n'est pas difficile mais nous ne pouvons pas dire que $\Omega$, lui, est un domaine de la forme simple.

Dans cet article, nous allons montrer que, au cas de $n=3$, on peut trouver des exemples de $\Omega$ et de $B$ en forme très simple pour lesquels le problème mixte (1.1) est mal posé au sens de $C^{\infty}$.

Théorème 1. Soit $\Omega$ un domaine de $\mathbf{R}^{3}$ compact et convexe dont la frontière $S$ est indéfiniment différentiable et contient

$$
S_{0}=\left\{x \in \mathbf{R}^{3} ; x_{1}^{2}+x_{2}^{2}=1,\left|x_{3}\right| \leqq 1\right\} \text {. }
$$

Etant donné $v(x)$ un vecteur réel indéfiniment différentiable défini sur $S$ vérifiant sur $S_{0}$ la condition suivante: 


$$
\left\{\begin{array}{l}
x_{1} v_{1}(x)+x_{2} v_{2}(x)=a \neq 0 \\
x_{1} v_{2}(x)-x_{2} v_{1}(x)=b \\
v_{3}(x)=c
\end{array}\right.
$$

où $a, b, c$ sont des constantes réelles. Alors, à la condition

$$
d(x)=d \quad \text { sur } \quad S_{0}
$$

pour d une constante réelle, le problème mixte (1.1) n'est pas bien posé au sens de $C^{\infty}$ si

$$
\sqrt{b^{2}+c^{2}}>d \geqq-\sqrt{b^{2}+c^{2}}, \quad d^{2}+c^{2} \neq 0 .
$$

D'après le théorème 1 il est immédiat de voir que pour l'opératcur frontière $B$ vérifiant (1.3), au cas de $d(x) \equiv 0$, le problème mixte (1.1) n'est pas bien posé au sens de $C^{\infty}$ si $c \neq 0$.

Comme on l'aperecevra tout de suite par la méthode de démonstration du théorème, le problème (1.1) est mal posé au sens de $C^{\infty}$ au cas que le domaine $\Omega$ soit convexe et que $\partial \Omega$ contienne un ouvert de $S_{0}$ où $B$ satisfait à (1.3), à (1.4) et à (1.5).

Nous allons démontrer le théorème 1 dans les paragraphes 2 et 3 . En ce moment là, la méthode de Ludwig [8] pour construire des solutions asymptotiques à unc caustique convexe joue un rôle essentiel.

Considérons le cas où $\Omega=\left\{x \in \mathbb{R}^{3} ; x_{1}^{2}+x_{2}^{2}<1\right\}$. S'il a lieu (1.3) et (1.4) pour tout $x_{3} \in \mathbb{R}$ on peut caractériser les constantes $\{a, b, c, d\}$ pour lesquelles le problème mixte (1.1) est bien posé. Nous le considérerons brièvement dans le paragraphe 4 .

§2. Construction des Solutions Asymptotiques de l'Équation des Ondes (d'après la méthode de Ludwig [8] pour l'expansion asymptotique uniforme à une caustique convexe)

D'abord introduisons l'espace $S^{p}(D)$. Etant donnés $D$ un domaine et $p$ un réel. $w(x ; k) \in S^{p}(D)$ signifie que pour tout $k \geqq 1 w(x ; k)$ appartient à $C^{\infty}(D)$ et que $\left\{k^{-p} w(x ; k) ; k \geqq 1\right\}$ est borné dans $C^{\infty}(D)$.

Notons que si $w_{j}(x ; k) \in S^{p_{J}}(D), j=0,1,2, \ldots$ et $\quad p_{0} \geqq p_{1} \geqq \cdots \geqq p_{j} \geqq$ $p_{J+1} \cdots \rightarrow-\infty$, on peut trouver $w \in S^{p_{0}}(D)$ telle que pour tout $j$ 


$$
w-\sum_{l=0}^{j-1} w_{l} \in S^{p_{J}}(D) \cdot{ }^{1)}
$$

Dès maintenant nous désignons un point de $\mathbb{R}^{3}$ par $(x, y, z)$ au lieu de $\left(x_{1}, x_{2}, x_{3}\right)$. Dans ce paragraphe en suivant Ludwig [8] nous cherchons une solution de l'équation des ondes sous la forme suivante:

$$
\begin{aligned}
u(x, y, z, t ; k)= & \exp \left\{i k\left(\theta(x, y)+\zeta z+\frac{z}{\sqrt{k}}-\tau t-\frac{1}{\sqrt{k}} \frac{\zeta}{\tau} t\right)\right\} \\
& \times\left\{g_{0}(x, y, z, t ; k) V\left(\rho(x, y) k^{\frac{2}{3}}\right)\right. \\
& \left.+g_{1}(x, y, z, t ; k) \frac{1}{i k^{\frac{1}{3}}} V^{\prime}\left(\rho(x, y) k^{2}\right)\right\}
\end{aligned}
$$

où $g_{i} \in S^{p}\left(\mathbf{R}^{4}\right), i=0,1, \zeta$ et $\tau$ sont des constantes réelles telles que

$$
\tau=\sqrt{1+\zeta^{2}}
$$

et $V(z)$ est la fonction d'Airy. ${ }^{2)}$

Nous prenons $\theta(x, y)$ et $\rho(x, y)$ des fonctions à valeurs réels indéfiniment différentiables de façon qu'il ait lieu

$$
\begin{aligned}
& \rho(x, y)=0 \quad \text { sur } \quad x^{2}+y^{2}=r_{0}^{2} \\
& (\nabla \theta)^{2}+\rho(\nabla \rho)^{2}=1
\end{aligned}
$$

où $\nabla=\left(\frac{\partial}{\partial x}, \frac{\partial}{\partial y}\right)$. Dès maintenant $\Delta$ signifie Laplacien par rapport à $(x, y)$, c'est-à-dire, $\frac{\partial^{2}}{\partial x^{2}}+\frac{\partial^{2}}{\partial y^{2}}$.

Notons que les fonctions vérifiant (2.3) (2.5) s'écrivent pour $(x, y)$, $x^{2}+y^{2} \geqq r_{0}^{2}$ comme suit: désignons par $(r, \omega)$ les coordonnées polaires de l'espace $(x, y)$, c'est-à-dire,

$$
\begin{aligned}
& x=r \cos \omega \\
& y=r \sin \omega,
\end{aligned}
$$

1) Voir, par exemple, Hörmander, le thćorème 2.7 de Pscudo-differential opcrators and hypoelliptic operators, Proc. Symp., on singular integrals, Chicago, 1966.

2) Voir, A. Erdélyi, Asymptotic expansions, Dover Publ., New York, 1965, page 94. 
alors quand $r \geqq r_{0}$

$$
\begin{aligned}
& \theta=\frac{r_{0}}{r} \omega \\
& \rho=\left[\frac{3}{2}\left\{\sqrt{r^{2}-r_{0}^{2}}-\operatorname{Arccos} \frac{r_{0}}{r}\right\}\right]^{\frac{2}{3}} .
\end{aligned}
$$

Posons

$$
\psi(x, y, z, t ; k)=k\left(0+\zeta z+\frac{1}{\sqrt{k}} z-\tau t-\frac{1}{\sqrt{k}} \frac{\zeta}{\tau} t\right) .
$$

$\Lambda$ ppliquons $\square$ à $u(x, y, z, t ; k)$ donnćc par (2.1) ct nous avons, en utilisant les propriétés de la fonction d'Airy

$$
\begin{gathered}
V^{\prime \prime}(z)+z V(z)=0 \\
V^{\prime \prime \prime}(z)+z V^{\prime}(z)+V(z)=0 \\
-\exp (-i \psi) \square u \\
=-k^{2} V g_{0}\left[(\nabla \theta)^{2}+\rho(\nabla \rho)^{2}\right] \\
-\frac{k^{\frac{5}{3}}}{i} V^{\prime} g_{1}\left[(\nabla \theta)^{2}+\rho(\nabla \rho)^{2}\right] \\
+i k^{\frac{5}{3}} V^{\prime} g_{0} 2[\nabla \theta \cdot \nabla \rho]+k^{\frac{4}{3}} V^{\prime \prime} g_{1} 2[\nabla \theta \cdot \nabla \rho] \\
+i k V\left[2 \nabla \theta \cdot \nabla g_{0}+\Delta \theta \cdot g_{0}+2 \rho \nabla \rho \cdot \nabla g_{1}\right. \\
+g^{\frac{2}{3}} V^{\prime}\left[2 \nabla \rho \cdot \nabla g_{0}+\Delta \rho \cdot g_{0}+2 \nabla \theta \cdot \nabla g_{1}+\Delta \theta \cdot g_{1}\right] \\
+V \cdot \Delta g_{0}+i k^{-\frac{1}{3}} V^{\prime} \Delta g_{1} \\
\left.+(i(k \zeta+\sqrt{k}))^{2}\left(g_{0} V+\frac{1}{i k^{\frac{1}{3}}} g\right)_{1}^{2} V_{1}\right] \\
+2 i(k \zeta+\sqrt{k})\left(\frac{\partial g_{0}}{\partial z} V+\frac{1}{i k^{\frac{1}{3}}} \frac{\partial g_{1}}{\partial z} V^{\prime}\right) \\
+
\end{gathered}
$$




$$
\begin{aligned}
& -\left(-i\left(k \tau+\sqrt{k} \frac{\zeta}{\tau}\right)\right)^{2}\left(g_{0} V+\frac{1}{i k^{\frac{1}{3}}} g_{1} V^{\prime}\right) \\
& +2 i\left(k \tau+\sqrt{k} \frac{\zeta}{\tau}\right)\left(\frac{\partial g_{0}}{\partial t} V+\frac{1}{i k^{\frac{1}{3}}} \frac{\partial g_{1}}{\partial t} V^{\prime}\right) \\
& +\left(\frac{\partial^{2} g_{0}}{\partial z^{2}}-\frac{\partial^{2} g_{0}}{\partial t^{2}}\right) V+\frac{1}{i k^{\frac{1}{3}}}\left(\frac{\partial^{2} g_{1}}{\partial z^{2}}-\frac{\partial^{2} g_{1}}{\partial t^{2}}\right) V^{\prime}
\end{aligned}
$$

Donc si l'on pose

$$
g_{i}(x, y, z, t ; k)=\sum_{j \geqq 0} k^{p-\frac{j}{2}} g_{i j}(x, y, z, t ; k) \quad i=0,1,
$$

$g_{i j} \in S^{0}\left(\mathbf{R}^{4}\right)$, grâce à (2.2), à (2.3), à (2.4) et à (2.5), pour que $\square u=0$ soit satisfait asymptotiquement, il suffit que $g_{i j}$ vérifient pour tout $j$

$$
\begin{aligned}
& 2 \nabla \theta \cdot \nabla g_{0 j}+\Delta \theta \cdot g_{0 j}+2 \rho \nabla \rho \cdot \nabla g_{1 j}+\rho \Delta \rho \cdot g_{1 j}+(\nabla \rho)^{2} g_{1 j} \\
+ & 2 \zeta \frac{\partial g_{0 j}}{\partial z}+2 \tau \frac{\partial g_{0 j}}{\partial t}+\frac{i}{\tau^{2}} g_{0 j} \\
+ & 2 \frac{\partial g_{0 j-1}}{\partial z}+2 \frac{\zeta}{\tau} \frac{\partial g_{0 j-1}}{\partial t}-i \square g_{0 j-2}=0 \\
& 2 \nabla \rho \cdot \nabla g_{0 j}+\Delta \rho \cdot g_{0 j}+2 \nabla \theta \cdot \nabla g_{1 j}+\Delta \theta \cdot g_{1 j} \\
+ & 2 \zeta \frac{\partial g_{1 j}}{\partial z}+2 \tau \frac{\partial g_{1 j}}{\partial t}+\frac{i}{\tau^{2}} \cdot g_{1 j} \\
+ & 2 \frac{\partial g_{1 j-1}}{\partial z}+2 \frac{\zeta}{\tau} \frac{\partial g_{1 j-1}}{\partial t}-i \square g_{1 j-2}=0 .
\end{aligned}
$$

Pour trouver $\left\{g_{0 j}, g_{1 j}\right\}$ successivement, d'abord considérons le système suivant:

$$
\begin{aligned}
& 2 \nabla \theta \cdot \nabla h_{0}+\Delta \theta \cdot h_{0}+2 \rho \nabla \rho \cdot \nabla h_{1}+\rho \Delta \rho \cdot h_{1}+(\nabla \rho)^{2} h_{1} \\
& \quad+2 \zeta \frac{\partial h_{0}}{\partial z}+2 \tau \frac{\partial h_{0}}{\partial t}+\frac{i}{\tau^{2}} h_{0}=f_{0}
\end{aligned}
$$

$$
\begin{gathered}
2 \nabla \rho \cdot \nabla h_{0}+\Delta \rho \cdot h_{0}+2 \nabla \theta \cdot \nabla h_{1}+\Delta \theta \cdot h_{1} \\
+2 \zeta \frac{\partial h_{1}}{\partial z}+2 \tau \frac{\partial h_{1}}{\partial t}+\frac{i}{\tau^{2}} h_{1}=f_{1} .
\end{gathered}
$$


En posant $H=\left\{h_{0}, h_{1}\right\}$ et $F=\left\{f_{0}, f_{1}\right\}$ nous désignons (2.10) et (2.11) par

$$
\mathscr{L} H=F
$$

Soit $\Gamma_{r_{0}}$ un cylindre $\left\{(x, y, z, t) ; x^{2}+y^{2}=r_{0}^{2}\right\}$. Etant donné un point $\left(x_{0}, y_{0}, z_{0}, t_{0}\right) \in \Gamma_{r_{0}}$. Une droite

$$
\left\{\left(x_{0}+\frac{y_{0}}{r_{0}} l, y_{0}-\frac{x_{0}}{r_{0}} l, z_{0}+\zeta l, t_{0}+\tau l\right) ;-\infty<l<\infty\right\}
$$

est tangente à $\Gamma_{r_{0}}$ en $\left(x_{0}, y_{0}, z_{0}, t_{0}\right)$.

Pour chaque point $P=(x, y, z, t)$ tel que $x^{2}+y^{2}>r_{0}^{2}$ il existe un et un seul point $\left(x_{0}, y_{0}, z_{0}, t_{0}\right)$ de $\Gamma_{r_{0}}$ tel qu'il a lieu pour certain $l<0$

$$
\left(x_{0}+\frac{y_{0}}{r_{0}} l, y_{0}-\frac{x_{0}}{r_{0}} l, z_{0}+\zeta l, t_{0}+\tau l\right)=(x, y, z, t) .
$$

Désignons ce point $\left(x_{0}, y_{0}, z_{0}, t_{0}\right) \in \Gamma_{r_{0}}$ par $Q(P)$ et la droite passant $P$ et $Q(P)$ par $L(P)$.

Quand on écrit $P=(r, \omega: z, t)$ il signifie que $(x, y)$ s'exprime en les coordonnées polaires, à savoir,

$$
P=(r \cos \omega, r \sin \omega, z, t) .
$$

Et nous décrivons aussi $g(x, y, z, t)$ par $g(r, \omega: z, t)$, c'est-à-dire,

$$
g(r, \omega: z, t)=g(r \cos \omega, r \sin \omega, z, t) .
$$

Pour $P=(r, \omega: z, t)$ et $W=\left(\omega_{0}, z_{0}, t_{0}\right)$ on désigne par $P_{j W}$ le point $\left(r, \omega-j \omega_{0}: z-j z_{0}, t-j t_{0}\right)$.

Pour $H=\left\{h_{0}, h_{1}\right\}$ posons sur $\rho(x, y) \geqq 0$

$$
H^{ \pm}=h_{0}(x, y, z, t) \pm \sqrt{\rho(x, y)} h_{1}(x, y, z, t) .
$$

Lemme 2.1. Soit $r_{1}>r_{0}$. Pour $H_{0}^{-}\left(r_{1}, \omega: z, t\right) \in C^{\infty}\left(\Gamma_{r_{1}}\right)$ quelconque il existe une fonction appartenant à $\left(C^{\infty}\left(\mathbb{R}^{4}\right)\right)^{2}$ avec les propriétés suivantes:

$$
\begin{aligned}
& H^{-}(x, y, z, t)=H_{0}^{-}(x, y, z, t) \quad \text { sur } \quad \Gamma_{r_{1}} \\
& \mathscr{L} H=F \quad \text { dans } \quad\left\{(x, y, z, t) ; x^{2}+y^{2} \geqq r_{0}^{2}\right\}
\end{aligned}
$$




$$
\begin{aligned}
\operatorname{supp} H & \cap\left\{(x, y, z, t) ; x^{2}+y^{2} \geqq r_{0}^{2}\right\} \\
& \subset\left\{L(P) ; P \in \operatorname{supp} H_{0}^{-} \cup \operatorname{supp} F \cap\{\rho \geqq 0\}\right\} .
\end{aligned}
$$

Et plus, si $F \equiv 0$, en posant

$$
W_{r}=\left(\varphi_{r}, \zeta\left(\sqrt{r^{2}-r_{0}^{2}}+\sqrt{r_{1}^{2}-r_{0}^{2}}\right), \tau\left(\sqrt{r^{2}-r_{0}^{2}}+\sqrt{r_{1}^{2}-r_{0}^{2}}\right)\right)
$$

où $\varphi_{r}=\operatorname{Arccos} \frac{r_{0}}{r_{1}}+\operatorname{Arccos} \frac{r_{0}}{r}$, pour tout $P=(r, \omega: z, t), r \geqq r_{0}$, on $a$

$$
H^{+}(P)=c\left(r, r_{1}, r_{0}\right) H_{0}^{-}\left(P_{W_{r}}\right)
$$

où $c\left(r, r_{1}, r_{0}\right)$ est une constante positive déterminée par $r, r_{0}$ et $r_{1}$.

Démonstration. Puisque pour $M$ une constante positive arbitraire, on a

$$
\frac{\partial \rho}{\partial r} \geqq c_{M}>0 \quad \text { si }|\rho| \leqq M
$$

nous pouvons prendre $\theta$ et $\rho$ comme un système des coordonnées de l'espace $(x, y)$. En utilisant $(2.5)$ on a

$$
\begin{aligned}
& \nabla \theta \cdot \nabla u=(\nabla \theta)^{2} \frac{\partial u}{\partial \theta} \\
& \nabla \rho \cdot \nabla u=(\nabla \rho)^{2} \frac{\partial u}{\partial \rho}
\end{aligned}
$$

Si l'on pose $\eta=\sqrt{\rho}$ dans $\left\{(x, y, z, t) ; x^{2}+y^{2} \geqq r_{0}^{2}\right\}$, on déduit de (2.10)

$$
\begin{aligned}
& 2(\nabla \theta)^{2} \frac{\partial h_{0}}{\partial \theta}+\Delta \theta \cdot h_{0}+2(\nabla \rho)^{2} \frac{\partial \eta h_{1}}{\partial \eta}+\eta \Delta \rho \cdot \eta h_{1} \\
+ & 2 \zeta \frac{\partial h_{0}}{\partial z}+2 \tau \frac{\partial h_{0}}{\partial t}+\frac{i}{\tau^{2}} h_{0}=f_{0} .
\end{aligned}
$$

Multiplier (2.11) par $\eta$ et on obtient

$$
\begin{aligned}
& 2(\nabla \rho)^{2} \frac{\partial h_{0}}{\partial \eta}+\eta \Delta \rho \cdot h_{0}+2(\nabla \theta)^{2} \frac{\partial \eta h_{1}}{\partial \theta}+\Delta \theta \cdot \eta h_{1} \\
+ & 2 \zeta \frac{\partial \eta h_{1}}{\partial z}+2 \tau \frac{\partial \eta h_{1}}{\partial t}+\frac{i}{\tau^{2}} \eta h_{1}=\eta f_{1} .
\end{aligned}
$$


Puisque $H^{ \pm}=h_{0} \pm \eta h_{1}$, de $(2.10)^{\prime}$ et de $(2.11)^{\prime}$ on déduit

$$
\begin{aligned}
& 2(\nabla \theta)^{2} \frac{\partial H^{ \pm}}{\partial \theta} \pm 2(\nabla \rho)^{2} \frac{\partial H^{ \pm}}{\partial \eta}+2 \zeta \frac{\partial H^{ \pm}}{\partial z}+2 \tau \frac{\partial H^{ \pm}}{\partial t} \\
+ & (\Delta \theta \pm \eta \Delta \rho) H^{ \pm}+\frac{i}{\tau^{2}} H^{ \pm}=F^{ \pm} .
\end{aligned}
$$

Rappelons que $\nabla \theta, \nabla \rho, \Delta \theta$ et $\Delta \rho$ sont des fonctions indéfiniment différentiables de $\theta$ et de $\eta^{2}$. D'après $(\nabla \rho)^{2} \geqq c_{M}>0$ pour tout $|\eta| \leqq$ $M^{\frac{1}{2}}$, si l'on prend

$$
\eta=\sqrt{\rho\left(r_{1} \cos \omega, r_{1} \sin \omega\right)} \quad\left(=\eta_{1}\right)
$$

comme le plan initial, $(2.18)^{-}$admet une solution unique $H^{-}(\eta, \theta, z, t)$ $(-\infty<\eta<\infty)$ pour la donnée initiale

$$
H^{-}\left(\eta_{1}, \theta, z, t\right)=H_{0}^{-}\left(r_{1}, \frac{r_{0}}{r} \theta: z, t\right)
$$

En tenant compte de la forme des coefficients de (2.18) et de $F^{ \pm}$, si l'on pose

$$
H^{+}(\eta, \theta, z, t)=H^{-}(-\eta, \theta, z, t)
$$

$H^{+}$vérifie $(2.18)^{+}$. Donc

$$
\begin{aligned}
& \tilde{h}_{0}(\eta, \theta, z, t)=H^{+}(\eta, \theta, z, t)+H^{-}(\eta, \theta, z, t) \\
& \tilde{h}_{1}(\eta, \theta, z, t)=\frac{1}{\eta}\left(H^{+}(\eta, \theta, z, t)-H^{-}(\eta, \theta, z, t)\right)
\end{aligned}
$$

satisfont à $(2.10)^{\prime}$ et à $(2.11)^{\prime}$. D'autre part il existe $h_{i}(\sigma, \theta, z, t) \in C^{\infty}\left(\mathbf{R}^{4}\right)$ telles que

$$
h_{i}\left(\eta^{2}, \theta, z, t\right)=\tilde{h}_{i}(\eta, \theta, z, t), \quad i=0,1
$$

Alors on voit immédiat que $H=\left\{h_{0}(\rho, \theta, z, t), h_{1}(\rho, \theta, z, t)\right\}$ satisfait à (2.14).

Notons que $(\nabla \theta \pm \sqrt{\rho} \nabla \rho, \zeta, \tau)$ est constant sur $L(P)$ parce que $(\nabla \theta \pm \sqrt{\rho} \nabla \rho)^{2}+\zeta^{2}=\tau^{2}$. Donc si $F \equiv 0$, puisque $H^{ \pm}$est une solution de

$$
2(\nabla \theta \pm \sqrt{\rho} \nabla \rho) \nabla H^{ \pm}+2 \zeta \frac{\partial H^{ \pm}}{\partial z}+2 \tau \frac{\partial H^{ \pm}}{\partial t}
$$




$$
+(\Delta \theta \pm \sqrt{\rho} \Delta \rho) H^{ \pm}+\frac{i}{\tau^{2}} H^{ \pm}=0
$$

(2.17) déduit de la définition de $H^{+}$.

Nous etendrons $H$ dans $\{\rho \leqq 0\}$ de sorte que $H$ appartienne à $C^{\infty}\left(\mathbf{R}^{4}\right)$. C'est possible parce que $H \in C^{\infty}\left(\mathbf{R}^{4} \cap\{\rho \geqq 0\}\right)$.

Etant données $G_{j 0}^{-}\left(r_{1}, \omega: z, t ; k\right) \in S^{0}\left(\Gamma_{r_{1}}\right), r_{1}>r_{0}, j=0,1,2, \ldots$ en appliquant le lemme 2.1 on peut obtenir successivement $G_{j}(x, y, z, t ; k)=$ $\left\{g_{0 j}, g_{1 j}\right\}$ de façon que

$$
G_{j}^{-}\left(r_{1}, \omega: z, t\right)=G_{j 0}^{-}\left(r_{1}, \omega: z, t\right)
$$

et qu'ils vérifient (2.8) et (2.9) pour $j=0,1, \ldots$, dans $\{\rho \geqq 0\}$ en posant $G_{-2} \equiv G_{-1} \equiv 0$. Donc il existe $G=\left\{g_{0}, g_{1}\right\} \in\left(S^{p}\left(\mathbf{R}^{4}\right)\right)^{2}$ telle que

$$
g_{i}(x, y, z, t ; k)-\sum_{j=0}^{N} k^{p-\frac{j}{2}} g_{i j}(x, y, z, t ; k) \in S^{p-\frac{N}{2}}\left(\mathbf{R}^{4}\right)
$$

Alors pour la fonction $u$ définie par (2.1) avec $\left\{g_{0}, g_{1}\right\}$ ci-dessus il a lieu

$$
\square u \in S^{-\infty}\left(\mathbf{R}^{4}\right)
$$

En effet, $\square u \in S^{-\infty}\left(\mathbf{R}^{4} \cap\{\rho \geqq 0\}\right)$ se déduit immédiat du fait que (2.8) et (2.9) sont vérifiés dans $\{\rho \geqq 0\}$.

Notons que pour tous $j \geqq 0$ et $N>0$ il existe une constante $C_{j N}>0$ telle que

$$
\left|\mathscr{L} G_{j}+2 \frac{\partial G_{j-1}}{\partial z}+2 \frac{\zeta}{\tau} \frac{\partial G_{j-1}}{\partial t}-i \square G_{j-2}\right| \leqq C_{j N}|\rho|^{N}
$$

Cela se déduit de $G_{j} \in C^{\infty}\left(\mathbf{R}^{4}\right)$ et de

$$
\mathscr{L} G_{j}+2 \frac{\partial G_{j-1}}{\partial z}+2 \frac{\zeta}{\tau} \frac{\partial G_{j-1}}{\partial t}-i \square G_{j-2}=0 \quad \text { dans }\{\rho \geqq 0\} .
$$

Notons que l'on a pour $\rho<0$

$$
V\left(\rho k^{\frac{2}{3}}\right)=\frac{1}{2} \frac{1}{\sqrt{\pi}}\left(-\rho k^{\frac{2}{3}}\right)^{-1 / 4} e^{-(2 / 3) \sqrt{-\rho} k}\left(1+0\left(\frac{3}{2}\left(-\rho k^{\frac{2}{3}}\right)^{-2 / 3}\right)\right)
$$

lorsque $k \rightarrow \infty$ (Erdélyi, page 94). En utilisant une estimation 


$$
\begin{gathered}
\left|e^{-\frac{2}{3^{2} /-\rho} k}(-\rho)^{l}\right|=\left|\left(\frac{2}{3} k\right)^{-2 l} e^{-\frac{2}{3} v^{\prime-\rho} k}\left(-\frac{2}{3} k \sqrt{-\rho}\right)^{2 l}\right| \\
\leqq k^{-2 l} C_{l},
\end{gathered}
$$

on obtient $\square u \in S^{-\infty}\left(\mathbf{R}^{4} \cap\{\rho \leqq 0\}\right)$ de la forme de $\square u$ et du comportement asymptotique de $V\left(\rho k^{\frac{2}{3}}\right)$. Donc (2.19) est démontré.

Si l'on utilise le comportement asymptotique de $V\left(\rho k^{\frac{2}{3}}\right)$ pour $\rho>0$ (Erdélyi, page 94) on obtient, en posant

$$
\begin{aligned}
& \varphi^{ \pm}=k\left(\theta \pm \frac{2}{3} \rho^{\frac{3}{2}}\right)+k \zeta z+\sqrt{k} z-k \tau t-\sqrt{k} \frac{\zeta}{\tau} t, \\
& u=\exp (i \psi)\left\{g_{0} V\left(\rho k^{\frac{2}{3}}\right)+\frac{1}{i k^{\frac{1}{3}}} g_{1} V^{\prime}\left(\rho k^{\frac{2}{3}}\right)\right\} \\
& =\frac{k^{-1 / 6}}{2 \sqrt{\pi}} e^{-\frac{\pi i}{4}}\left\{e^{i \varphi^{+}}\left(Z^{+}+\frac{1}{k} \tilde{Z}^{+}\right)\right. \\
& \left.+e^{i \varphi^{-}+\frac{\pi i}{2}}\left(Z^{-}+\frac{1}{k} \tilde{Z}^{-}\right)\right\} \\
& =u^{+}+u^{-},
\end{aligned}
$$

où

$$
Z^{ \pm}=\rho^{-\frac{1}{4}}\left(g_{0} \pm \sqrt{\rho} g_{1}\right)=\rho^{-\frac{1}{4} G^{ \pm}}
$$

et $\tilde{Z}^{ \pm} \in S^{p}\left(\mathbf{R}^{4}\right)$. Par la même sorte nous avons

$$
\begin{gathered}
u_{j}=\exp (i \varphi)\left(g_{0 j} V+\frac{1}{i k^{\frac{1}{3}}} g_{1 j} V^{\prime}\right) \\
=\frac{k^{-\frac{1}{6}}}{2 \sqrt{\pi}} e^{-\frac{\pi}{4} i}\left\{e^{i \varphi^{+}}\left(Z_{j}^{+}+\frac{1}{k} \tilde{Z}_{j}^{+}\right)\right. \\
\left.+e^{i \varphi^{-}+\frac{\pi i}{2}}\left(Z_{j}^{-}+\frac{1}{k} \tilde{Z}_{j}^{-}\right)\right\} \\
=u_{j}^{+}+u_{j}^{-} .
\end{gathered}
$$

Alors il est évident que 


$$
u^{ \pm}=\sum_{j \geqq 0} k^{p-\frac{j}{2}} u_{j}^{ \pm} \quad \text { asymptotiquement }
$$

Supposons que $0<r_{0}<1$ et $\zeta$ sont des constantes rélles vérifiant

$$
-a \sqrt{1-r_{0}^{2}}+b r_{0}+c \zeta-d \sqrt{1+\zeta^{2}}=0 .
$$

Etant donnée

$$
\begin{aligned}
m(\omega: z, t ; k) & =\exp \left\{i k\left(r_{0} \omega+\zeta z+\frac{1}{\sqrt{k}} z-\tau t-\frac{1}{\sqrt{k}} \frac{\zeta}{\tau} t\right)\right\} \\
& \times \sum_{j \geqq 0} k^{q-\frac{j}{2}} m_{j}(\omega: z, t ; k)
\end{aligned}
$$

$m_{j}(\omega: z, t ; k) \in S^{0}\left(\Gamma_{1}\right)$. Supposons que

$$
\operatorname{supp} m_{j} \subset\left\{(\omega, z, t) ; t \geqq t_{0}\right\} .
$$

Nous allons construire une fonction $u(x, y, z, t ; k)$ telle que

$$
\begin{aligned}
& \square u \in S^{-\infty}\left(\mathbf{R}^{4}\right) \\
& B u^{-}-m \in S^{-\infty}\left(\Gamma_{1}\right) \\
& \operatorname{supp} u \subset\left\{(x, y, z, t) ; t \geqq t_{0}\right\} .
\end{aligned}
$$

D'abord notons que

$$
\left.\frac{\partial \varphi^{ \pm}}{\partial r}\right|_{r=1}= \pm \sqrt{1-r_{0}^{2}},\left.\quad \frac{\partial \varphi^{ \pm}}{\partial \omega}\right|_{r=1}=r_{0} .
$$

D'après (2.20), (2.22) et (2.29)

$$
\begin{aligned}
\left.B u^{-}\right|_{r=1}= & \frac{1}{2 \sqrt{\pi}} k^{-1 / 6} e^{-\frac{\pi i}{4}} \exp \left(i \varphi^{-}\right) \\
& \times \sum_{j=0}^{\infty} k^{p-\frac{j}{2}}\left[\left\{i k\left(-a \sqrt{1-r_{0}^{2}}+b r_{0}+c \zeta-d \tau\right)\right.\right. \\
& \left.\left.+i \sqrt{k}\left(c-\frac{d \zeta}{\tau}\right)\right\}\left(Z_{j}^{-}+\frac{1}{k} \tilde{Z}_{j}^{-}\right)+\left(B Z_{j}^{-}+\frac{1}{k} B \tilde{Z}_{j}^{-}\right)\right]
\end{aligned}
$$

Donc on obtient (2.27) pourvu que 


$$
p-\frac{1}{6}+\frac{1}{2}=q
$$

et que

$$
\begin{array}{r}
\left.i\left(c-d \frac{\zeta}{\tau}\right) Z_{j}^{-}\right|_{r=1}=2 \sqrt{\pi} e^{i \pi / 4}\left\{m_{j}-i\left(c-d \frac{\zeta}{\tau}\right)\left(B Z_{j-1}^{-}\right.\right. \\
\left.\left.+B \tilde{Z}_{\bar{j}-3}+\tilde{Z}_{j-2}\right)\right\}
\end{array}
$$

pour $j=0,1,2, \ldots$ Supposons

$$
c-d \frac{\zeta}{\tau} \neq 0
$$

Alors puisque

$$
\left.G_{j}^{-}\right|_{r=1}=\left.\rho^{\frac{1}{4}} Z_{j}^{-}\right|_{r=1}
$$

nous pouvons trouver en récurrence $G_{j}=\left\{g_{0 j}, g_{1 j}\right\}, j=0,1,2, \ldots$ vérifiant (2.8), (2.9) et (2.31). Alors on voit immédiat que $u(x, y, z, t ; k)$ définie par (2.1) avcc les $G_{j}$ construites au-dessus satisfait à (2.26), à (2.27) et à (2.28).

D'après (2.20)

$$
\begin{aligned}
\left.B u^{+}\right|_{r=1}= & \exp \left(i \varphi^{+}\right) \sum_{j=0}^{\infty} k^{q-\frac{i+1}{2}} \mid\left\{i k\left(a \sqrt{1-r_{0}^{2}}+b r_{0}+c \zeta-d \tau\right)\right. \\
& \left.\left.+i \sqrt{k}\left(c-d \frac{\zeta}{\tau}\right)\right\}\left(Z_{j}^{+}+\frac{1}{k} \tilde{Z}_{j}^{+}\right)+\left(B \tilde{Z}_{j}^{+}+\frac{1}{k} B \tilde{Z}_{j}^{+}\right)\right]
\end{aligned}
$$

Donc on a pour $k \rightarrow \infty$

$$
\left.B u^{+}\right|_{r=1} \sim \frac{1}{2 \sqrt{\pi}} \exp \left(i \varphi^{+}\right) k^{q+\frac{1}{2}} 2 i a \sqrt{1-r_{0}^{2}} e^{-\frac{\pi i}{4}} Z_{0}^{+}
$$

D'autre part en appliquant à $G_{0}$ le résultat (2.17)

$$
\begin{aligned}
Z_{0}^{+}(P) & =c\left(r_{0}\right) Z_{0}^{-}\left(P_{W}\right) \\
& =c\left(r_{0}\right)\left\{i\left(c-d \frac{\zeta}{\tau}\right)\right\}^{-1} 2 \sqrt{\pi} e^{\frac{\pi i}{4}} m_{0}\left(P_{W}\right)
\end{aligned}
$$

où 


$$
W=\left(2 \operatorname{arc} \cos r_{0}, 2 \zeta \sqrt{1-r_{0}^{2}}, 2 \tau \sqrt{1-r_{0}^{2}}\right)
$$

Donc nous avons

Proposition 2.2. Soit

$$
B=a \frac{\partial}{\partial r}+b \frac{\partial}{\partial \omega}+c \frac{\partial}{\partial z}+d \frac{\partial}{\partial t} .
$$

Etant données $r_{0}, \zeta$ des constantes réelles telles que $0<r_{0}<1$ et que

$$
-a \sqrt{1-r_{0}^{2}}+b r_{0}+c \zeta-d \sqrt{1+\zeta^{2}}=0 .
$$

Prenons une fonction de $C^{\infty}\left(\Gamma_{1}\right)$ de la forme

$$
\begin{aligned}
& m(\omega: z, t ; k)=\exp \left\{i k\left(r_{0} \omega+\zeta z+\frac{z}{\sqrt{k}}-\tau t-\frac{1}{\sqrt{k}} \frac{\zeta}{\tau} t\right)\right\} \\
& \times k^{q} n(\omega: z, t ; k),
\end{aligned}
$$

où $n \in S^{0}\left(\Gamma_{1}\right)$ et $n-n_{0}(\omega: z, t) \in S^{-\frac{1}{2}}$ pour une certaine fonction $n_{0}(\omega$ : $z, t) \in C^{\infty}\left(\Gamma_{1}\right)$.

Alors il existe une fonction $u(x, y, z, t ; k)$ de la forme (2.1) satis-

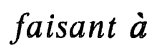

$$
\begin{aligned}
& \square u \in S^{-\infty}\left(\mathbf{R}^{4}\right) \\
& {\left[B u^{-}-m\right]_{r=1} \in S^{-\infty}\left(\Gamma_{1}\right)}
\end{aligned}
$$

et $\grave{a}$

$$
\operatorname{supp} u \cap\{\rho \geqq 0\} \subset \underset{P \in \operatorname{supp} m}{\cup} L(P)
$$

Et plus

$$
\begin{aligned}
\left.B u^{+}\right|_{r=1}=\exp \{ & \left.i k\left(r_{0} \omega+\zeta z+\frac{z}{\sqrt{k}}-\tau t-\frac{1}{\sqrt{k}} \frac{\zeta}{\tau} t\right)\right\} \\
& \times k^{q+\frac{1}{2}} n_{1}(\omega: z, t ; k)
\end{aligned}
$$

où $n_{1} \in S^{0}\left(\Gamma_{1}\right)$ et

$$
n_{1}(P)-c\left(r_{0}\right)\left(-2 a \sqrt{1-r_{0}^{2}}\right)\left(c-d \frac{\zeta}{\tau}\right)^{-1} n_{0}\left(P_{W}\right) \in S^{-\frac{1}{2}}\left(\Gamma_{1}\right)
$$




\section{§3. La Démonstration du Théorème 1}

Soit $\Omega_{1}=\left\{(x, y, z, t) ; x^{2}+y^{2}<1\right\}$. Nous nous plaçons sous les hypothèses de la proposition 2.2 sur l'opérateur frontière $B$ et des constantes $r_{0}$ et $\zeta$.

Etant donnée

$$
\begin{aligned}
m(\omega: z, t ; k)=\exp & \left\{i k\left(r_{0} \omega+\zeta z+\frac{z}{\sqrt{k}}-\tau t-\frac{1}{\sqrt{k}} \frac{\zeta}{\tau} t\right)\right\} \\
\times & n(\omega: z, t),
\end{aligned}
$$

où $n(\omega: z, t) \in C^{\infty}\left(\Gamma_{1}\right)$ telle que

$$
\operatorname{supp} n(\omega: z, t) \subset\left\{(\omega, z, t) ; 0 \leqq t \leqq \varepsilon_{0}\right\}
$$

$$
n\left(0: 0, \varepsilon_{0} / 2\right)=1
$$

Appliquons la proposition 2.2 à $m(\omega: z, t ; k)$ de (3.1). Alors il existe $u_{0}(x, y, z, t ; k)$ satisfaisant à

$$
\begin{aligned}
& \square u_{0} \in S^{-\infty}\left(\bar{\Omega}_{1}\right) \\
& {\left[B u_{0}^{-}-m\right]_{r=1} \in S^{-\infty}\left(\Gamma_{1}\right)} \\
& \left.B u_{0}^{+}\right|_{r=1}=\exp \left(i \varphi^{+}\right)\left(k^{\frac{1}{2}} m_{0}+\tilde{m}_{0}\right),
\end{aligned}
$$

où

$$
\begin{aligned}
& m_{0}(P)=c_{0} n\left(P_{W}\right), \\
& c_{0}=-c\left(r_{0}\right) 2 a \sqrt{1-r_{0}^{2}}\left(c-d \frac{\zeta}{\tau}\right)^{-1}
\end{aligned}
$$

et

$$
\tilde{m}_{0} \in S^{0}\left(\Gamma_{1}\right)
$$

Nous avons

$$
\operatorname{supp} u_{0} \cap \Omega_{1} \subset \Omega_{1} \cap\left\{0 \leqq t \leqq 2 \tau \sqrt{1-r_{0}^{2}}+\varepsilon_{0}\right\}
$$

et 


$$
\left.\operatorname{supp} B u_{0}^{+}\right|_{r=1} \subset\left\{2 \tau \sqrt{1-r_{0}^{2}} \leqq t \leqq 2 \tau \sqrt{1-r_{0}^{2}}+\varepsilon_{0}\right\} .
$$

Appliquons encore la proposition 2.2 à $-\left[B u_{0}^{+}\right]_{r=1}$. Il existe une fonction $u_{1}$ vérifiant

$$
\begin{aligned}
& \square u_{1} \in S^{-\infty}\left(\bar{\Omega}_{1}\right) \\
& {\left[B u_{1}^{-}+B u_{0}^{+}\right]_{r=1} \in S^{-\infty}\left(\Gamma_{1}\right)}
\end{aligned}
$$

et

$$
\left[B u_{1}^{+}\right]_{r=1}=\exp \left(i \varphi^{+}\right)\left(k m_{1}+\tilde{m}_{1}\right)
$$

où

$$
\begin{aligned}
& m_{1}(P)=c_{0}^{2} n\left(P_{2 W}\right), \\
& \tilde{m}_{1} \in S^{\frac{1}{2}}\left(\Gamma_{1}\right) .
\end{aligned}
$$

Et nous avons aussi

$$
\begin{aligned}
& \operatorname{supp} u_{1} \cap \Omega_{1} \subset \Omega_{1} \cap\left\{2 \tau \sqrt{1-r_{0}^{2}} \leqq t \leqq 4 \tau \sqrt{1-r_{0}^{2}}+\varepsilon_{0}\right\} \\
& \operatorname{supp}\left[B u_{1}^{+}\right]_{r=1} \subset\left\{4 \tau \sqrt{1-r_{0}^{2}} \leqq t \leqq 4 \tau \sqrt{1-r_{0}^{2}+} \varepsilon_{0}\right\}
\end{aligned}
$$

En répétant cette procédure nous obtenons $u_{j}, j=1,2, \ldots$ avec les propriétés suivantes:

$$
\begin{aligned}
& \square u_{j} \in S^{-\infty}\left(\bar{\Omega}_{1}\right) \\
& {\left[B u_{j-1}^{+}+B u_{j}^{-}\right]_{r=1} \in S^{-\infty}\left(\Gamma_{1}\right)} \\
& {\left[B u_{j}^{+}\right]_{r=1}=\exp \left(i \varphi^{+}\right)\left(k^{\frac{j+1}{2}} m_{j}+\tilde{m}_{j}\right)}
\end{aligned}
$$

où

$$
\begin{gathered}
m_{j}(P)=c_{0}^{j+1} n\left(P_{(j+1) W}\right), \\
\tilde{m}_{j} \in S^{\frac{j}{2}}\left(\Gamma_{1}\right), \\
\operatorname{supp} u_{j} \cap \Omega_{1} \subset\left\{2 j \tau \sqrt{1-r_{0}^{2}} \leqq t \leqq 2(j+1) \tau \sqrt{1-r_{0}^{2}}+\varepsilon_{0}\right\} .
\end{gathered}
$$

Fixons $\varepsilon_{0}$ de sorte que $0<\varepsilon_{0}<\tau \sqrt{1-r_{0}^{2}}$. Définissons $u(x, y, z, t ; k)$ par 


$$
u=\sum_{j=0}^{\infty} u_{j}
$$

C'est bien possible parce que pour tout $(x, y, x, t) \in \bar{\Omega}_{1}$ au plus deux $u_{j}$ ne sont pas nulles. On voit immédiat

$$
\begin{aligned}
& \square u \in S^{-\infty}\left(\bar{\Omega}_{1}\right) \\
& {[B u-m]_{r=1} \in S^{-\infty}\left(\Gamma_{1}\right)} \\
& \operatorname{supp} u \cap \Omega_{1} \subset\{t \geqq 0\} .
\end{aligned}
$$

D'autre part dans un voisinage de

$$
P_{j}=\left(1,(j+1) \varphi_{0}: 2(j+1) \zeta \sqrt{1-r_{0}^{2}}, 2(j+1) \tau \sqrt{1-r_{0}^{2}}\right)
$$

u s'exprime

$$
u=u_{j+1}^{-}+\sum_{l \leqq j} u_{l}
$$

Et plus

$$
\begin{gathered}
u_{j+1}^{-} \sim \exp \left(i \varphi^{-}\right) k^{\frac{j}{2}} c_{0}^{j+1} m_{j}(P) \\
\left|\sum_{l=0}^{j} u_{l}\right| \leqq C k^{\frac{j-1}{2}}
\end{gathered}
$$

Donc on obtient grâce à (3.3) et à (3.4)

$$
\sup _{\Omega_{1} \cap\left\{0 \leqq t \leqq 2(j+1) \tau \sqrt{1-r_{0}^{2}}+\varepsilon_{0}\right\}}|u(x, y, z, t ; k)| \geqq c_{0}^{j+1} k^{\frac{j}{2}} .
$$

Donc nous avons

Proposition 3.1. Supposons que $0<r_{0}<1$ et $\zeta$ vérifient

$$
-a \sqrt{1-r_{0}^{2}}+b r_{0}+c \zeta-d \sqrt{1+\zeta^{2}}=0
$$

Alors pour une fonction $m(\omega: z, t ; k)$ donnée par (3.1), (3.2) et (3.3) on peut trouver une fonction $u(x, y, z, t ; k)$ satisfaisant à

$$
\begin{aligned}
& \square u \in S^{-\infty}\left(\bar{\Omega}_{1}\right) \\
& {[B u-m]_{r=1} \in S^{-\infty}\left(\Gamma_{1}\right)}
\end{aligned}
$$




$$
\operatorname{supp} u \cap \Omega_{1} \subset\{t \geqq 0\}
$$

et $\grave{a}$

$$
\sup _{\Omega_{1} \cap\left[0, t_{0}\right]}|u(x, y, z, t ; k)| \geqq\left(c_{0} k^{\frac{1}{2}}\right)^{\left(\frac{t_{0}}{2 r^{\prime}-\zeta^{2} \sqrt{1-r_{0}^{2}}}-1\right)}
$$

Nous nous mettons à démontrer le théorème 1 annoncé dans l'introduction. Avant tout, admettons le lemme suivant, dont la démonstration n'est pas difficile.

Lemme 3.2. Supposons que des constantes réelles $b, c$ et $d$ vérifient

$$
\sqrt{b^{2}+c^{2}}>d \geqq-\sqrt{b^{2}+c^{2}}, \quad d^{2}+c^{2} \neq 0 .
$$

Si $b \cdot d \geqq 0$, pour tout $n$ entier positif on peut trouver des constantes réelles $r_{n}$ et $\zeta_{n}$ telles que

$$
\begin{aligned}
& 1-\frac{1}{n}<r_{n}<1 \quad\left(1-\frac{1}{n}<-r_{n}<1 \text { si } b \cdot d<0\right) \\
& -a \sqrt{1-r_{n}^{2}}+b r_{n}+c \zeta_{n}-d \sqrt{1+\zeta_{n}^{2}}=0 \\
& c-d \frac{\zeta_{n}}{\sqrt{1+\zeta_{n}^{2}}} \neq 0 .
\end{aligned}
$$

Supposons que le problème mixte (1.1) soit bien posé au sens de $C^{\infty}$ sous la condition (1.5). Alors d'après la définition pour toute $\{m$, $f\} \in C_{0}^{\infty}\left(S \times\left(0, t_{0}\right]\right) \times C_{0}^{\infty}\left(\bar{\Omega} \times\left(0, t_{0}\right]\right)$ il existe une solution unique $u(x, y$, $z, t) \in C^{\infty}\left(\bar{\Omega} \times\left[0, t_{0}\right]\right)$ vérifiant

$$
\begin{array}{lll}
\square u=f & \text { dans } & \Omega \times\left[0, t_{0}\right] \\
B u=m & \text { sur } \quad & S \times\left[0, t_{0}\right] \\
u(x, y, z, 0)=\frac{\partial u}{\partial t}(x, y, z, 0)=0
\end{array}
$$

et pour tous $t \in\left[0, t_{0}\right]$ et $\alpha \in\{0,1,2, \ldots\}^{4}$ il existe des constantes $C_{\alpha}, N_{\alpha}$ telles que

$$
\sup _{\bar{\Omega} \times[0, t]}\left|D^{\alpha} u(x, y, z, t)\right| \leqq C_{\alpha}\left\{|m|_{N, S \times[0, t]}+|f|_{N, \bar{\Omega} \times[0, t]}\right\}
$$


où

$$
|m|_{N, S \times[0, t]}=\sup _{\substack{|\alpha| \leq N \\(x, y, z, t) \in S \times[0, t]}}\left|D^{\alpha} m\right| .
$$

D'après (1.5) le lemme 3.2 est applicable. On voit que $\left\{\zeta_{n} ; n=\right.$ $1,2, \ldots\}$ est borné et $\zeta_{n} \rightarrow \zeta_{\infty}$ lorsque $n \rightarrow \infty$. Supposons que pour tout $n$

$$
1-\frac{1}{n}<r_{n}<1
$$

Quand $1-\frac{1}{n}<-r_{n}<1$ il suffit de remplacer les rôle de $u^{+}$et de $u^{-}$ dans les considérations jusqu'à la proposition 3.1.

Prenons $t_{0}=\frac{1}{2}$ et $N=N_{(0,0,0,0)}$. Choisissons $n$ de façon que

$$
\frac{1 / 2}{2 \sqrt{1-r_{n}^{2}} \sqrt{1+\zeta_{n}^{2}}}-1>2 N+1
$$

cela est possible puisque $r_{n} \rightarrow 1, \zeta_{n} \rightarrow \zeta_{\infty}$ lorsque $n \rightarrow \infty$. Avec cet $n$ définissons une fonction $m$ par

$$
\begin{gathered}
m(\omega: z, t ; k)=\exp \left\{i k\left(r_{n} \omega+\zeta_{n} z+\frac{z}{\sqrt{k}}-\tau_{n} t-\frac{1}{\sqrt{k}} \frac{\zeta_{n}}{\tau_{n}} t\right)\right\} \\
\times n(\omega: z, t ; k)
\end{gathered}
$$

où

$$
\operatorname{supp} n \subset\left\{(\omega, z, t) ; 0<t<\tau_{n} \sqrt{1-r_{n}^{2}},|z|<\left|\zeta_{n}\right| \sqrt{1-r_{n}^{2}}\right\}
$$

En tenant compte de la définition de $S$ nous pouvons considérer $m(\omega: z, t ; k)$ comme une fonction définie sur $S \times[0, \infty)$. L'application de la proposition 3.1 à $m(\omega: z, t ; k)$ nous donne l'existence d'une fonction $u(x, y, z, t ; k)$ telle que

$$
\begin{aligned}
& \square u \in S^{-\infty}\left(\bar{\Omega}_{1}\right) \\
& B u-m \in S^{-\infty}\left(\Gamma_{1}\right) \\
& \operatorname{supp} u \cap \Omega_{1} \subset\{t \geqq 0\}
\end{aligned}
$$

et 


$$
\sup _{\Omega_{1} \cap[0,1 / 2]}|u(x, y, z, t ; k)| \geqq\left(c_{0} k^{\frac{1}{2}}\right)\left(\frac{1 / 2}{2 v^{\prime} 1-r_{n}^{2} r^{\prime} 1+\zeta_{n}^{2}}-1\right) .
$$

Si l'on applique (2.32) à chaque $u_{j}$ apparue en construction de $u$, on voit que pour tout $t \in\left[0,2(j+1) \tau_{n} \sqrt{1-r_{n}^{2}}\right]$

$$
\operatorname{supp}_{(x, y, z)} u(x, y, z, t ; k) \subset\left\{(x, y, z) ; x^{2}+y^{2} \leqq 1,|z|<2(j+1) \zeta_{n} \sqrt{1-r_{n}^{2}}\right\}
$$

Donc pour $0 \leqq t \leqq 1 / 2$,

$$
\operatorname{supp}_{(x, y, z)} u(x, y, z, t ; k) \subset\left\{(x, y, z) ; x^{2}+y^{2} \leqq 1,|z|<\frac{1}{2}\right\} .
$$

Donc quand on considère $u(x, y, z, t ; k)$ commc une fonction définie dans $\Omega \times[0, \infty)$ nous avons de (3.9) et de (3.10)

$$
\left\{\begin{array}{l}
\square u \in S^{-\infty}(\bar{\Omega} \times[0,1 / 2]) \\
{[B u-m]_{S} \in S^{-\infty}\left(S \times\left[0, \frac{1}{2}\right]\right)} \\
u(x, y, z, 0 ; k)=\frac{\partial u}{\partial t}(x, y, z, 0 ; k)=0 .
\end{array}\right.
$$

Grâce à la forme de $m(\omega: z, t ; k)$ on a $|m|_{N, S \times[0,1]} \leqq C_{N} k^{N}$, ct en conséquence

$$
|B u|_{N, S \times[0,1]} \leqq C_{N} k^{N}
$$

Et on déduit de (3.12)

$$
|\square u|_{N, \bar{\Omega} \times[0,1]} \leqq C_{N} \cdot
$$

La substitution de (3.13) et de (3.14) à (3.6) nous donne

$$
\sup _{\Omega \times\left[0, \frac{1}{2}\right]}|u(x, y, z, t ; k)| \leqq C_{N} k^{N} .
$$

D'autre part, d'après (3.11)

$$
\sup _{\Omega \times\left[0, \frac{1}{2}\right]}|u(x, y, z, t ; k)| \geqq\left(c_{0} k^{\frac{1}{2}}\right)\left(\frac{1 / 2}{2 v^{\prime} \frac{1-2}{1-r_{n}^{2}} v^{\prime} \frac{\zeta_{n}^{2}}{1}}-1\right)
$$

par (3.7) 


$$
\geqq c_{0}^{\prime} k^{N+\frac{1}{2}} \quad\left(c_{0}^{\prime}>0\right) .
$$

Donc on a

$$
c_{0}^{\prime} k^{N+\frac{1}{2}} \leqq C_{N} k^{N} \quad \text { pour tout } k \geqq 1
$$

C'est une contradiction. Donc on a démontré le théorème 1.

\section{§4. Remarque}

La considération que nous avons faite pour démontrer le théorème 1 csi essentiellement pour le domaine $\Omega=\left\{(x, y, z) ; x^{2}+y^{2}<1\right\}$. Si nous nous bornons au cas de $\Omega$ au-dessus et de $B$ de la forme

$$
B=a \frac{\partial}{\partial r}+b \frac{\partial}{\partial \omega}+c-\frac{\partial}{\partial z}+d \frac{\partial}{\partial t}
$$

où $a, b, c$ et $d$ sont des constantes réelles et $a>0$, il est possible de déterminer quand (1.1) est bien posé au sens de $C^{\infty}$. A savoir,

Théorème 2. (i) $S i \sqrt{b^{2}+c^{2}} \leqq d$ (1.1) est bien posé au sens de $L^{2}$.

(ii) Si $\sqrt{b^{2}+c^{2}}>d \geqq-\sqrt{b^{2}+c^{2}}, d^{2}+c^{2} \neq 0$, (1.1) est mal posé au sens $C^{\infty}$.

(iii) Si $c=d=0$, pour tout $b(1.1)$ est bien posé au sens de $C^{\circ}$.

(iv) Si $d \neq-1$ et $-\sqrt{b^{2}+c^{2}}>d \geqq-\sqrt{b^{2}+c^{2}+1}$, (1.1) est bien posé au sens de $C^{x}$.

(v) Si $d=-1$ ou $d<-\sqrt{b^{2}+c^{2}+1}$ (1.1) esi mal posé au sens de $C^{\infty}$.

En effet, (i) est un cas spécial des résultats de Miyatake [9].

(ii) est le théorème 1 lui-même.

(iii) peut être montré par le même raisonnement d'Ikawa [3], qui est pour $\Omega \subset \mathbb{R}^{2}$.

(iv) est l'un des exemples plus typiques appartenant à la classe introduite dans Ikawa [6].

Au cas de (v) on trouve $r, \zeta$ récls et $\operatorname{Im} \tau<0$ tels que

$$
-a \sqrt{\tau^{2}-r^{2}-\zeta^{2}}+b r+c \zeta-d \tau=0 .
$$


Alors la méthode de construction des solutions asymptotiques de Lax [7] est profitable et on peut démontrer sans aucune difficulté le fait que (1.1) est mal posé au sens de $C^{\infty}$.

\section{Références}

[1] Erdélyi, A., Asymptotic expansions, Dover Publ., New York, 1965.

[2] Ikawa, M., On the mixed problem for the wave equation with an oblique derivative boundary condition, Proc. Japan Acad., 44 (1968), 1033-1037.

[3] Ikawa, M., Mixed problem for the wave equation with an oblique derivative boundary condition, Osaka J. Math., 7 (1970), 495-525.

[4] Ikawa, M., Remarques sur les problèmes mixtes pour l'équation des ondes, Colloque international C.N.R.S., (1972) astérisque 2 et 3, 217-221.

[5] Ikawa, M., Remarques sur les problèmes mixtes pour l'équation des ondes, à paraître.

[6] Ikawa, M., Problèmes mixtes pas nécessairement $L^{2}$-bien posés pour les équations strictement hyperboliques, à paraître.

[7] Lax, P. D., Asymptotic solutions for oscillatory initial value problems, Duke Math. J., 24 (1957), 627-646.

[8] Ludwig, D., Uniform asymptotic expansions at a caustic, Comm. Pure Appl. Math., 19 (1966), 215-250.

[9] Miyatake, S., Mixed problem for hyperbolic equation of second order, J. Math. Kyoto Univ., 13 (1973) 435-487. 